\title{
IL RITORNO DELLE FRONTIERE INTERNE IN EUROPA E LA SOLIDARIETÀ AI MIGRANTI IN TRANSITO: IL CASO DELLA VAL ROJA
}

\author{
The return of internal borders in Europe and solidarity \\ with migrants in transit: the case of the Roya Valley
}

Luca Giliberti*

\begin{abstract}
Riassunto. II presente articolo analizza la solidarietà ai migranti in transito nell'Europa della "crisi dell'accoglienza" attraverso lo studio di caso della Val Roja, una piccola valle francese al confine con I'Italia. II contributo contestualizza il ritorno delle frontiere interne in Europa e si focalizza sulle forme, gli attori e le pratiche della solidarietà ai migranti in questa valle. Si tratta di una solidarietà endogena, che nasce in particolare dalle reti di neorurali e si struttura attorno a valori condivisi, oltreché ad un determinato approccio di difesa del territorio. Allo stesso tempo, voci ostili ai migranti e alla solidarietà emergono, all'interno di un conflitto sociale tra i nativi - le cosiddette familles de souches - e i più recenti abitanti. L'articolo si basa su una ricerca etnografica di un anno e mezzo, svolta attraverso un processo di immersione nella realtà locale e l'uso di tecniche quali l'osservazione partecipante e le interviste semi-strutturate.
\end{abstract}

Parole chiave: frontiera; migrazione di transito; crisi dell'accoglienza; solidarietà; neoruralità;

\begin{abstract}
This article analyzes solidarity with migrants in transit in Europe at the time of the "reception crisis " through the case study of the Roya Valley, a small French valley on the border with Italy. The contribution contextualises the return of internal borders in Europe and it focuses on the forms, actors and practices of solidarity with migrants in this valley. This is an endogenous solidarity, which arises in particular from the neorural networks and it is structured around common values, as well as a specific approach to defense of the territory. At the same time, hostile voices towards migrants and solidarity emerge, within a social conflict between natives - the socalled familles de souche - and the more recent inhabitants. The article is based on a one and a half year ethnographic research, through a process of immersion in the local reality, using tecniques as participatory observation and semi-structured interviews.
\end{abstract}

Keywords: border; transit migration; reception crisis; solidarity; neorurality.

\footnotetext{
Università degli Studi di Genova; Université Côte d'Azur-URMIS. Genova, Italia. E-mail: lucagiliberti@hotmail.com. Orcid:0000-0002-8413-1109.
} 


\section{Il ritorno dei confini europei visto dalla Val Roja}

La Val Roja, piccola valle alla frontiera tra I'Italia e la Francia, è oggi costituita da cinque paesi francesi (Breil-sur-Roya, Saorge, Fontan, La Brigue e Tende); tale configurazione è recente, considerato che negli ultimi 160 anni la frontiera si è spostata per ben due volte, evidenziando la provvisorietà e la mobilità dei confini'. Lungo i 59 Km del fiume Roja, la valle comincia e termina in Italia; dal Colle di Tenda - varco alpino alla quota di 1.871 metri, che separa le Alpi Liguri dalle Alpi Marittime - arriva fino al mare nella città di Ventimiglia. I cinque villaggi, che contano in totale circa 6.000 abitanti, sono circondati dal territorio italiano a nord, a sud e a est. Per andare a Nizza o genericamente verso la Francia, la strada più corta e più veloce è l'autostrada, il cui casello più vicino è proprio Ventimiglia; nella città italiana, inoltre, i valligiani sono soliti recarsi, per esempio, per realizzare le proprie compere. La valle, negli ultimi anni, con la chiusura della frontiera franco-italiana, si ritrova al centro delle rotte migratorie verso il Nord Europa (Giliberti, 2020).

La convenzione di Schengen, applicata dalla metà degli anni '90, sancisce l'indurimento proibizionista dei confini esterni - nella costruzione di una sigillata "Fortezza Europa" - stabilendo allo stesso tempo la fine dei controlli alle frontiere interne. Le due misure si costruiscono, in qualche modo, l'una come presupposto dell'altra; la libertà di movimento all'interno dello spazio europeo viene giustificata, in effetti, proprio in rapporto alla chiusura esterna. Tale chiusura delle frontiere esterne modifica lo scenario delle migrazioni internazionali, facendo emergere nuove categorie migratorie in relazione alla produzione di nuovi dispositivi di gestione dei flussi e di discorsi per legittimarli (Streiff-Fénart, Poutignat, 2008).

Il concetto di "transito" si impone così nel linguaggio delle organizzazioni internazionali per riferirsi a un nuovo tipo di mobilità ("migrazioni di transito"), nonché a un dispositivo materiale per il blocco o il respingimento dei migranti clandestini ("centri di transito"); tali concetti emergenti sono euristicamente rilevanti non tanto in relazione a ciò che indicano - persone in transito, soggetti che si trovano in un luogo di passaggio - quanto rispetto al quadro istituzionale e normativo che motiva tale creazione lessicale, a partire dal divieto di circolazione Sud/Nord. II concetto di "Transit migration" (Hess, 2012) legata al dispositivo Schengen e alla crescita del "border control" (Collyer, Düvell, de Hass, 2010), si associa in questo senso alle nozioni di proibizionismo migratorio e migrazione irregolare (Düvell, 2012; Düvell, Molodikova, Collyer, 2014).

\footnotetext{
Nel 1860, quando I'Italia non esisteva ancora, i villaggi oggi francesi della Val Roja appartenevano al Regno Savoia del Piemonte e della Sardegna, insieme a Nizza. Dal 1860 Breil-Sur-Roya, Saorge e ciò che oggi è Fontan - comune indipendente dal 1871 - diventano francesi; dopo la Seconda Guerra Mondiale, nel 1947, anche Tende e La Brigue passano sotto la giurisdizione della Francia.
} 
A partire dal 2015, con i controlli sistematici e la militarizzazione delle frontiere interne, il concetto di "transit migrant" comincia ad essere applicato nel linguaggio corrente anche ai confini intraeuropei, allo stesso modo di come si costruiva negli anni '90 in relazione alle frontiere esterne (Giliberti, Queirolo Palmas, 2020). In termini di immagine, da una "Fortezza Europa", sigillata all'esterno e aperta all'interno, si passa ad uno spazio chiuso sia all'esterno che all'interno, in cui è l'intera Europa a prendere la forma di un "borderland" (Balibar, 2009).

Da quasi cinque anni a questa parte, con i controlli sistematici e la militarizzazione del territorio alla frontiera franco-italiana - come su diversi altri confini interni all'Europa ${ }^{2}$, giustificati dall'allarme terrorismo - si genera un blocco strutturale dei migranti in transito nello spazio europeo, per cui migliaia di persone provenienti da paesi come Eritrea, Sudan, Etiopia o Costa d'Avorio, si arenano in situazioni di marginalità nell'attesa di riuscire a passare un confine come quello franco-italiano. L'espressione "ritorno della frontiera interna" si riferisce alla reintroduzione dei controlli sistematici tra gli Stati europei, in una sorta di ritorno alla situazione pre-Schengen, anche se con un dispositivo di militarizzazione del territorio molto più importante. La Fortezza Europa, dunque, nella contemporanea ristrutturazione di Schengen, si presenta oggi come un reticolo in cui i controlli sistematici, oltreché ai margini esterni, si effettuano anche al suo interno.

Una serie di associazioni denunciano sin dal principio i controlli messi in atto alla frontiera franco-italiana in quanto discriminatori, strutturati sul racial profiling - definiti in francese contrôles au faciès -, basati sul colore della pelle e sulle caratteristiche fenotipiche (Gisti-Cimade-Adde-Anafé, 2015; Amnesty International, 2017; Anafé, 2019). Tali controlli razziali, che sono selettivi e svolgono funzione di filtro, coinvolgono determinati soggetti e non altri a partire da una logica razziale. Le violazioni di diritti alla frontiera, testimoniati e denunciati ormai da anni da associazioni e osservatori nazionali e internazionali, divengono parte costituente dei contemporanei governo delle migrazioni e regime di frontiera.

I controlli sistematici in territorio francese sono realizzati fino a $20 \mathrm{Km}$ dal confine e dunque prendono forma anche una volta varcato il territorio statale (Gachet-Deuzeide, 2018); in questo senso, da linea, la frontiera diventa banda larga. Una volta bloccati, la pratica generalizzata delle forze dell'ordine nei confronti dei migranti irregolari è quella del respingimento a Ventimiglia - 0 deportazione express (Aris Ascarcena, 2018) - che la maggior parte dei soggetti,

\footnotetext{
2 Per una mappa interattiva sul ritorno delle frontiere nello spazio Schengen, si veda: <https:// www.touteleurope.eu/actualite/schengen-la-carte-des-controles-aux-frontieres-nationales. html>.
} 
prima di riuscire a passare, sperimenta più volte. Si tratta di una sorta di gioco dell'oca, in cui si entra in Francia, si viene respinti a Ventimiglia, si riprova, si viene respinti di nuovo, si riprova ancora, fino a che la gran maggior parte riesce nell'intento. Dati dell'Anafé (2019), frutto di monitoraggi sul territorio, testimoniano come le persone provino fino a quindici volte a passare la frontiera prima di riuscire; in ogni caso, in maggiore o minor tempo, gran parte dei migranti riesce a passare e, secondo i dati di RRDP (2017), il 73\% delle persone accampate dichiara di essere bloccato a Ventimiglia da meno di 3 mesi. Ci sono anche persone che muoiono cercando di passare la frontiera tra l'Italia e la Francia: le cifre relative ai cadaveri ritrovati alla frontiera sud - senza poter sapere quanti siano quelli non ritrovati - si aggirano ad oggi attorno a trenta: schiacciati da camion e auto in autostrada e da treni nei tunnel, elettrificati sui tetti dei treni, annegati nel fiume Roja dopo essere precipitati da sentieri di montagna.

Nel tentativo di varcare il confine, i migranti tentano l'attraversamento di montagne e territori rurali, disegnando, in ogni caso, nuove rotte di transito nell'Europa del proibizionismo migratorio (Queirolo Palmas, 2017). Prendono forma così delle contro-mappe, facilitate nei diversi territori dalle pratiche di ospitalità e sostegno ai migranti in transito, che divengono in qualche modo elementi costitutivi delle rotte. Uno degli elementi chiave di questo nuovo scenario risulta essere l'irrompere sulla scena pubblica della società civile, che prende posizione (Fontanari, Borri, 2017) all'interno di un campo di battaglia (Ambrosini, 2018). Nelle diverse aree attraversate e lungo le diverse rotte del transito, in effetti, le reti solidali convivono con i movimenti anti-migranti (Rea et alii, 2019).

Sulla frontiera franco-italiana si assiste, sin dal 2015, all'irrompere di una solidarietà verso i migranti bloccati al confine (Giliberti, Queirolo Palmas, 2020), che convive con un altro segmento della popolazione che è invece contraria e denuncia l'azione solidale. In Val Roja una parte degli abitanti si espone in prima persona nelle reti della solidarietà, attraverso pratiche di ospitalità, cura, supporto al transito, sostegno alle rotte, oltreché in attività di denuncia pubblica della frontiera. L'eterogenea rete solidale include pratiche che tengono insieme la dimensione dell'azione politica con il sostegno umanitario; sono registri, questi, che nella "crisi dell'accoglienza" (Lendaro, Rodier, Vertongen, 2019) divengono sempre più interconnessi e presentano importanti spazi di ibridazione (Gerbier-Aublanc, 2018). Tali pratiche contribuiscono a costruire una contro-mappa dell'Europa che sfida il proibizionismo migratorio interno ai confini dell'Unione, aprendo rifugi e rotte invisibili per favorire il diritto alla libera circolazione.

Il presente contributo nasce da una ricerca etnografica basata sull'immersione, la partecipazione alla vita del territorio e la costruzione di un "fare comune" con i diversi attori del campo (Queirolo Palmas, Stagi, 
2017) ${ }^{3}$. Le principali tecniche impiegate sono l'osservazione partecipante in molteplici contesti della vita di valle e la raccolta di fonti orali, composte da ventinove interviste semi-strutturate, oltreché innumerevoli conversazioni informali riportate nei diari di campo; parte integrante del dispositivo di ricerca è inoltre un breve documentario sulle pratiche di solidarietà sul territorio ${ }^{4}$. Nel presente contributo si ripercorrono gli elementi fondamentali delle esperienze di solidarietà della valle e le caratteristiche delle reti che aiutano i migranti in transito.

\section{La solidarietà ai migranti in transito in un territorio rurale}

Alcuni solidali della valle cominciano ad essere attivi nel sostegno verso i migranti bloccati in frontiera sin dal giugno 2015, partecipando all'esperienza del Presidio Permanente No-Borders ${ }^{5}$ di Ventimiglia. Anne ${ }^{6}$, che fin da subito diventa uno dei soggetti chiave della solidarietà di valle, tornando dalle vacanze trova un messaggio di una sua amica nella segreteria del cellulare: "Volevo parlare con te per vedere cosa possiamo fare per i migranti..." (Anne, 57 anni, impiegata). Come lei, altre persone della valle si accorgono che da alcuni giorni erano stati ristabiliti i controlli sistematici alla frontiera e decidono di essere protagonisti di una solidarietà locale, prima sul confine e, in seguito, quando i migranti cominceranno a passare in Val Roja, sul proprio territorio.

Definiamo solidarietà l'insieme variegato di azioni al sostegno dei migranti, con alla base diverse motivazioni, registri e pratiche di riferimento, che non preveda una contropartita economica e sia estraneo al sistema formale di accoglienza (Giliberti, Queirolo Palmas, 2020). I solidali possono essere privati cittadini, organizzarsi in gruppi informali o in associazioni, e situarsi su diversi posizionamenti intermedi tra i poli di un approccio umanitario e politico; detto in altri termini, un approccio più assistenziale legato a risolvere mancanze delle politiche istituzionali, o un approccio più

3 La ricerca è stata realizzata nel quadro della Tesi di Dottorato in Sociologia (2016-2020) "Una valle di frontiera. Percorsi neorurali, mobilitazioni sociali e solidarietà ai migranti in Val Roja", in co-tutela Italia - Francia (Università degli Studi di Genova - Université Côte d'Azur/URMIS di Nizza). Il lavoro sul campo è stato realizzato dal gennaio 2017 al giugno 2018.

4 Il documentario "Transiti. Una valle solidale", nato dalla presente ricerca, realizzato insieme a Massimo Cannarella e prodotto dal Laboratorio di Sociologia Visuale dell'Università di Genova, è liberamente visionabile al link: < https://www.youtube.com/watch?v=YyRToR6j5_4> .

5 La prima esperienza di solidarietà ai migranti alla frontiera di Ventimiglia si struttura attorno al Presidio Permanente No-Borders, subito dopo il ripristino dei controlli nel giugno 2015 e il conseguente blocco di centinaia di persone che si insediano materialmente di fronte alla frontiera con Mentone. II Presidio è un campo auto-gestito, che unisce attivisti e transitanti, in cui viene allestita una cucina, sono creati bagni e docce, installati connessioni internet e alimentatori solari per ricaricare i telefoni. Gli accampati chiamano questo spazio la Bolla, un luogo protetto in cui "le regole di quest'Europa non entrano" (Martini, Palidda, 2018, p. 5) e in cui si discute, si organizza e si agisce fattivamente contro la frontiera e per la libertà di circolazione.

6 I nomi degli intervistati sono fittizi, per garantire il loro anonimato. 
contestatario e con una visione di società di fondo, apertamente critico verso il modello dominante, che pratica supporto al transito e attacca esplicitamente la gestione migratoria e il regime di frontiera contemporanei.

Alcuni autori, per fare riferimento all'eterogeneità dell'universo della solidarietà, propongono l'espressione "umbrella notion", perché copre - come un ombrello - un ampio spettro di registri d'azione, di attori e di pratiche (Birey et alii, 2019). Il concetto di solidarietà, come nota Kymlicka (2015), è stato finora utilizzato soprattutto per riferirsi ad attori prossimi, normalmente all'interno delle "comunità immaginate", come la famiglia o la nazione (Anderson, 2018). L'uso che si fa di questo concetto in relazione alla contemporanea "crisi dell'accoglienza" (Lendaro, Rodier, Vertongen, 2019) si estende invece a coloro i quali, come i migranti in transito, sono rappresentati in quanto outsiders nel discorso dominante in Europa.

La solidarietà della Val Roja - essenzialmente una risposta del territorio rurale al blocco dei migranti alla frontiera che cominciano a transitare per la valle - prende forma attraverso tre ambiti di azione, correlati ed interconnessi. Il primo ambito si riferisce alla solidarietà sul territorio in cui sono bloccati, e comprende la distribuzione del cibo e di beni di prima necessità, l'ospitalità diffusa in case e terreni private; in tale ambito può rientrare, inoltre, l'attività di avvistamento dei migranti in valle e del loro recupero e presa in carico, prima che vengano fermati dalla polizia e respinti a Ventimiglia. Un secondo ambito di azione si riferisce al sostegno al transito, prevedendo il supporto al passaggio di frontiera o semplicemente, dopo essere riusciti ad arrivare in Francia in modo autonomo, un appoggio per continuare il proprio percorso migratorio e/o presentare una domanda d'asilo in Francia. Un terzo ambito d'azione si costruisce sull'azione di monitoraggio della violenza della frontiera, sull'analisi del non rispetto delle leggi da parte dello Stato e sulla denuncia giuridica e politica. A geometrie variabili, la solidarietà si muove su questi tre ambiti, strettamente complementari e assolutamente non escludenti. Non è allora inusuale trovare degli attori che si impegnano contemporaneamente nel primo e nel secondo ambito, ma non nel terzo, o altri che si muovono nel primo ambito ma non nel secondo; altri ancora che operano nel terzo, spesso occupandosi anche degli altri due, ma non necessariamente.

Un'attività basata a Ventimiglia, che ha visto una forte partecipazione degli abitanti della valle, è quella della preparazione e distribuzione del cibo, malgrado un'ordinanza municipale che vietasse tale attività per ragioni formali di carattere igienico-sanitario, rappresentando un chiaro esempio di criminalizzazione dell'azione umanitaria ${ }^{7}$. Dipendendo dai periodi, i pasti

Tale ordinanza municipale è simile ad omologhe ordinanze di divieto di distribuzione di beni di prima necessità, che nel contesto proibizionista hanno preso forma in altri luoghi, ad esempio a 
preparati sono stati da poco meno di cento a qualche centinaio, fino agli 800-900 in periodi determinati, come l'estate 2017. I solidali della Val Roja, coordinati attorno all'associazione Roya Citoyenne - che riceve doni e finanzia l'acquisto delle materie prime - per circa un anno hanno garantito i pasti in maniera continuativa con una struttura rotatoria dei propri gruppi di villaggio; un giorno settimanale fisso per ogni gruppo di paese, con il supporto delle valli vicine (Val Bevera, Val Vesubia, ecc.). I dati raccolti da Roya Citoyenne parlano di 150.000 pasti preparati e serviti da valligiani solo nel 2017, quando poi vengono rimpiazzati dai solidali internazionali di Kesha Niya ${ }^{8}$.

Dall'estate 2016 la rotta dei migranti bloccati in frontiera comincia a passare dalla Val Roja con un'importante intensità e molti abitanti si sentono costretti a confrontarsi con la situazione sul proprio territorio; tornando a casa vedono migranti feriti ai bordi delle proprie strade e intervengono con un approccio spontaneista. Percependo una sorta di obbligo morale nel non poter lasciare per strada persone in difficoltà, alcuni cominciano a portarli in casa propria (Giliberti, 2018; Lendaro, 2018).

La gente si è sentita obbligata a prendere una posizione... quando sei in macchina, stai guidando e vedi qualcuno che cammina da due giorni, in piena estate quando fa un caldo mortale o in pieno inverno quando c'è la neve e lui indossa sandali e pantaloncini corti, non puoi non fare nulla... (Jeff, 61 anni, operatore nei servizi alla persona)

Se ci sono persone disastrate in enorme difficoltà ai bordi delle strade sei obbligato a fermarti, prenderle e portarle a casa tua...questa azione si fa spontaneamente, non puoi fare altrimenti... (Michel, 33 anni, contadino)

In valle, c'è una parte della popolazione che la pensa in questi termini e comincia la pratica dell'ospitalità diffusa nelle case private e in un terreno agricolo messo a disposizione da un agricoltore alternativo della valle, Cédric Herrou, divenuto paladino mediatico della causa. Tale ospitalità viene coordinata dall'associazione Roya Citoyenne ${ }^{9}$, con l'obiettivo di agglutinare

Parigi (Queirolo Palmas, 2017). A Ventimiglia tale misura, che ha ricevuto critiche da molteplici fronti, viene introdotta nell'agosto 2015 e revocata una prima volta nel maggio 2016; reintrodotta nell'agosto 2016, viene definitivamente revocata nell'aprile 2017, rimanendo in vigore per un totale di 19 mesi.

8 Kesha Niya Kitchen - che significa No problem in curdo - è un gruppo di attivisti denominati in valle $i$ Vichinghi, perché la maggior parte sono tedeschi. I vichinghi - che insieme ad altre attività di supporto ai migranti, sono specializzati nella preparazione e distribuzione del cibo in luoghi di frontiera - han lavorato nel campo di Grande-Synthe, nei pressi di Calais, prima di collaborare con Roya Citoyenne alla frontiera franco-italiana. Nel gennaio 2020, momento in cui si scrive, il gruppo è ancora attivo nella distribuzione del cibo a Ventimiglia.

9 "Roya Citoyenne" (https://www.roya-citoyenne.fr/) è un'associazione nata nel 2011 intorno alla mobilitazione contro l'assorbimento della valle alla CARF ("Communauté d'agglomération de la Riviera française") e per la difesa di un'inter-municipalità della Val Roja. Nel maggio 2016 i suoi statuti vengono modificati per dedicarsi alla questione migratoria, quando una serie di cittadini, già attivi nel sostegno verso migranti, decidono di coordinarsi per poter organizzare collettivamente le azioni di solidarietà. 
e organizzare la solidarietà che cominciava a prendere forma. Una parte importante della solidarietà di valle si esprimerà attorno all'associazione, ma diverse altre esperienze di sostegno ai migranti - reti più piccole, informali e, per scelta, meno mediatizzate ${ }^{10}$ - prendono ugualmente forma sul territorio in modo attivo senza essere legate alla piattaforma associativa.

La valle, da luogo rurale marginale e periferico, diviene un luogo centrale per le rotte migratorie verso la Francia e il Nord Europa. In particolare, dalla primavera fino alla fine dell'estate del 2017, decine di migranti entrano in valle ogni giorno. Nella primavera 2017, in effetti, in seguito alla prima condanna da parte del Tribunale di Nizza per violazione del diritto d'asilo del prefetto delle Alpi Marittime, i solidali ottengono un protocollo non scritto che sancisce temporaneamente il diritto di far passare i transitanti dai check points o dalla stazione ferroviaria - previo invio di una lista nominativa al fine di permettere loro di depositare una domanda d'asilo a Nizza. Tale corridoio legale facilita l'aumento massivo dei transitanti per quella rotta e migliaia di persone passano dal camping di Cédric Herrou, che diviene nella realtà un campo autogestito per migranti in transito. Nel giro di pochi mesi lo Stato invalida l'accordo raggiunto e chiude nuovamente il passaggio verso la domanda di asilo in Francia; I'accordo informale, nel corso degli anni viene poi ripristinato e invalidato molteplici volte, a seconda dei livelli contingenti di pressione migratoria e di gestione della stessa.

Risulta impossibile stabilire precisamente quanti migranti siano passati e si siano fermati di passaggio nella valle, in ogni caso diverse migliaia. Solo sul terreno agricolo di Breil sono 2.500 i soggetti transitati, con picchi di 250 persone contemporaneamente nell'estate 2017. Più di un centinaio di case solidali, dislocate in tutti i paesini, ospitano nel corso del tempo decine di migranti, in certi casi anche alcune centinaia. Nel gennaio 2017 nel paese di Saorge, in cui durante l'anno vivono circa 400 abitanti, vengono ospitate contemporaneamente una sessantina di persone in case private, ossia quasi il $20 \%$ della popolazione presente in quel momento nel paese. Sono numeri che, se considerati in relazione ad una valle di circa 6.000 abitanti, risultano sorprendenti. Anne, già citata precedentemente, molto attiva nell'organizzazione dell'ospitalità sul territorio, afferma con gli occhi sbarrati: "è arrivato un momento in cui non sapevamo più dove metterli...e cercavamo dappertutto persone che fossero disposte ad ospitarli...".

${ }^{10}$ La questione della mediatizzazione, trattata nel dettaglio in altri contributi (Giliberti, 2020), è oggetto di dibattito e di divisione all'interno delle reti solidali, perché implica una pressione politica e l'ottenimento di certi risultati per la rivendicazione in atto, ma al tempo stesso facilita l'aumento del processo di militarizzazione e riduce la facilità del passaggio di frontiera dei transitanti. 
La rete dei solidali è ben coordinata e, una volta che qualcuno avvista dei migranti in valle, in maniera rapida qualcun altro - in prossimità del posto - viene contattato telefonicamente affinché vada a prenderli prima che li individui la polizia e vengano così ospitati. In un territorio militarizzato, i solidali intercettano i transitanti che arrivano - a piedi, per i sentieri di montagna o per i binari ferroviari, in alcuni casi in bus o in treno, o accompagnati dai passeurs o dagli stessi solidali - e cercano di prenderli sotto la propria protezione prima che li trovi la polizia e li riporti a Ventimiglia con un refus d'entrée.

leri sera, girando per la valle, in chiesa a Breil ci imbattiamo in un ragazzino eritreo, che presentava alcune ferite ai piedi e non aveva idea di dove andare. Ci parla in tigrino e l'unica cosa che capiamo è che era venuto a piedi da Ventimiglia e che vorrebbe andare a Parigi. La custode della chiesa stava per buttarlo fuori e in strada a Breil sarebbe stato visto dalla polizia e riportato a Ventimiglia. Telefoniamo a Christian e gli spieghiamo la situazione; passano a malapena cinque minuti e una ragazza con sua figlia piccola appaiono per prendere in custodia il ragazzo. "Per il momento gli preparo un pasto caldo, dice, poi vedremo come ci organizziamo con lui e come facciamo per farlo arrivare a Parigi. Contatteremo gente che sta in posti di passaggio per vedere se possiamo farlo arrivare gradualmente", ci dice la ragazza; auguriamo in bocca al lupo al ragazzino eritreo e ci rallegriamo che questa notte la passerà in una calda casa di Breil e non in strada al freddo a Ventimiglia. Ci rallegriamo, inoltre, che il suo percorso possa continuare, anche grazie ai contatti che i solidali hanno e stanno costruendo con la loro azione. (Estratto del diario di campo, febbraio 2017)

Un'infrastruttura di transito si genera, composta da reti territoriali sparse in connessione tra di loro - come quella di Ventimiglia, della Valle del Loup, Marsiglia o Parigi -, che ricorda la ferrovia sotterranea degli Stati Uniti del XIX secolo (Foner, 2015). Una rete sotterranea di case solidali e approdi sicuri - che permetteva agli schiavi neri in fuga dalle piantagioni di risalire il Continente americano verso il nord fino ad approdare in Canada - si ripropone oggi in Europa, dove persone e reti dei territori attraversati facilitano il dispiegarsi delle rotte migranti (Queirolo Palmas, Rahola, 2020). Le azioni degli abolizionisti americani di ieri fanno eco a quelle dei solidali europei di oggi.

La solidarietà che prende forma nella valle, essenzialmente espressione di una parte dei suoi abitanti, risponde dunque ad una solidarietà endogena, che si compone di circa 150-200 famiglie, attorno al 10\% della popolazione. La solidarietà del territorio si declina in chiave intima, ospitando in casa propria, ma anche in chiave politica, rivendicando nuove politiche migratorie e nuovi modelli di società; tale dimensione dell'"ospitalità" si differenzia dal concetto di "accoglienza" - o di mancata accoglienza - che fa riferimento ad una dimensione istituzionale (Babels, 2019). L'esperienza di Roya Citoyenne, insieme a quella del campo di Breil11, sono accompagnate da altre esperienze

11 L'esperienza del campo, dapprima legata all'associazione Roya Citoyenne, si lega ben presto all'associazione Défends Ta Citoyenneté, che Cédric Herrou e collaboratori fondano ad hoc nel settembre 2017. 
di solidarietà attiva, più ridotte e volutamente meno mediatiche e visibili, come sottolinea Stephane.

Roya Citoyenne è un po' l'associazione, il portale mediatico... che gestisce il lato giuridico... e finanzia anche le diverse attività, visto che riceve molti doni... e poi ci sono molte altre persone che prendono altre iniziative, che giustamente hanno il desiderio di restare discreti, di non comunicare nei media, niente foto... ma fare le loro piccole storie nel loro angolo. $\mathrm{Ci}$ sono stati dei grandi dibattiti in Roya Citoyenne con quelli che volevano fare delle cose ma in modo anonimo, discreto, tranquillo... e quelli che dicevano: "No, bisogna parlarne, bisogna far venire i giornalisti, bisogna far smuovere le cose dal punto di vista politico..." I due punti di vista sono rispettabili... io penso che servono entrambi... sono visioni complementari... c'è una vetrina e poi piccole reti che fanno le proprie storie... (Stephane, 48 anni, commerciante e guida di montagna)

Se le persone implicate nella solidarietà attiva sono intorno al $10 \%$ della valle, di gran lunga più ampio è il numero dei soggetti che, senza essere nell'azione, sono in qualche modo a favore del sostegno verso i migranti. Tali abitanti possono partecipare in occasioni alle collette o donazioni, mettere in atto piccole e semplici azioni di solidarietà diretta, per esempio preparare/ comprare un pasto.

Forse più della metà della gente della valle è a favore, o per lo meno non è contro... gente che non si impegna tantissimo, ma può fare una telefonata, può dare due soldi per la causa, o può preparare o comprare un panino ad un migrante che passa, ed è, così alla leggera, a favore... quelli che sono contro sono di meno, la maggior parte delle persone magari non sono attive nell'azione ma sono a favore di ciò che facciamo... (Claire, 66 anni, farmacista in pensione)

\section{I diversi posizionamenti della popolazione di una valle: $\mathrm{i}$ souche e i neorurali nella "crisi dell'accoglienza"}

La Val Roja vive negli ultimi decenni due fenomeni demografici opposti ma paralleli e complementari, I'uno presupposto dell'altro. Da un lato, l'esodo rurale di una parte delle famiglie native - o familles de souche ${ }^{12}-$, dall'altro

12 In francese la parola souche presenta diverse sfumature di significato. Nel senso figurato in cui qui si usa - associato al concetto di famiglia - significa "серро" in termini di origine o di stirpe. Una famille de souche è dunque una famiglia autoctona, che rivendica legami storici con uno specifico territorio. Conserva lo stesso significato di "ceppo" quando si riferisce a un albero, definendo la parte dell'albero che rimane radicata alla terra quando il tronco viene abbattuto. Può significare anche "matrice" quando si parla, per esempio, di un biglietto o di un assegno ("la souche d'un chèque"), o, in senso figurato, della matrice culturale di uno scrittore o di un artista ("la souche culturelle d'un ecrivain où d'un artiste"). Quest'ultimo riferimento alla matrice è importante, perché associa il concetto a un universo culturale, come è il caso delle categorie di abitanti qui discusse. Oltreché usato in termini emici per riferirsi alle famiglie autoctone di un territorio, il concetto souche si utilizza, in termini nazionalistici e sovranisti, per riferirsi ai "Françaises de souche" - espressione molto usata da Jean Marie Le Pen - ossia i cosiddetti francesi di pura ceppa. Analisi recenti (Loperfido, 2017) dimostrano come nuove forme di razzismo si approprino della categoria souche per la propria riconfigurazione discorsiva contro l'immigrazione e per il rafforzamento di una presunta identità ancestrale. 
I'arrivo di una popolazione neorurale (Nogué, 2016) - in generale proveniente dalla classe media urbana francese, definiti come hippies dalle famiglie native - alla ricerca di una vita alternativa in campagna. Le due popolazioni costruiscono due universi culturali di riferimento, in contrapposizione su molte questioni, che generano parimenti dei conflitti sul territorio. All'interno delle reti di solidarietà una presenza dominante è quella dei neorurali, caratterizzata da una scelta politica e ideologica ben definita, ispirata dai principi della decrescita (Latouche, 2006; Rahbi, 2014). La quasi totalità delle persone che fanno parte delle reti di solidarietà non sono nate in Val Roja da famiglie autoctone, ma ci sono arrivate per scelta volontaria all'interno del proprio percorso biografico; sono giunti a partire dalla fine dagli anni '70, in una scelta di vita che, in una serie di casi, sono ispirate ai "sentieri dell'utopia anticapitalista" (Fréméaux, Jordan, 2012).

Se osservi i membri di Roya Citoyenne o anche delle nostre reti più informali e meno mediatizzate, vedrai che queste persone, salvo qualche caso, sono neorurali o discendenti di neorurali... Sono persone che sono venute qua con dei valori, che hanno salvaguardato e hanno trasmesso ai loro figli... Si tratta di gente che si è radicata in Val Roja e ha consolidato certi valori, che stanno poi alla base del sostegno ai migranti. Si tratta in primo luogo di una questione di valori e di credenze. Qui in valle le persone che si fanno espressione di questi valori hanno relazioni e connessioni tra di loro più forti che in altri luoghi, perché siamo meno numerosi, ci vediamo spesso, e si generano delle reti socioculturali e amicali che sono forse più forti di quelle della città... (Marcel, 55 anni, guida di montagna)

La scelta di vita ispirata a valori comunitari e alternativi dei neorurali è senza dubbio elemento chiave per la comprensione dell'approccio solidale nei confronti dei migranti (Mollard, 2017). Una questione di valori, dunque, come suggerisce Marcel, di stile di vita e di approcci alternativi.

Sicuramente la composizione neorurale influisce... persone che hanno scelto di venire a vivere qui perché è una valle naturalisticamente interessante, c'è poco turismo... persone che hanno scelto uno stile di vita... ciò determina un'apertura grande di solidarietà... di dire: non si lascia nessuno indietro. La composizione della popolazione mi sembra la spiegazione più chiara... (Marta, 30 anni, disoccupata)

Chi è cresciuto in posti di vita alternativi è chiaro che produce una società diversa... per fortuna che in certe campagne, con certi stili di vita alternativi, si muove qualcosa... io pure mi sono spesso interrogato sul perché la campagna in certi casi produce maggiore solidarietà... (Filippo, 34 anni, operatore nei servizi alla persona)

Tanto Marcel come Marta e Filippo ci parlano di reti composte da persone che hanno scelto di abitare quel contesto e ne sono divenute protagoniste, partecipando alle vicende e alla costruzione identitaria del territorio, con cui costruiscono una forte identificazione. Sono le stesse persone che partecipano attivamente alle lotte cittadine per la difesa del proprio territorio, dalla rivendicazione per il mantenimento della linea ferroviaria locale e dei servizi pubblici, come la posta e le scuole della valle, sino alle mobilitazioni contro la 
grande opera a forte impatto ambientale del doppio tunnel di Tenda o quella dell'appartenenza imposta alla CARF (Communauté d'agglomération de la Riviera française) - un'inter-municipalità amministrativa di costa e non di valle, pensata per il turismo e non per una vita rurale sostenibile -, come si analizza in profondità in ulteriori contributi (Giliberti, 2020).

I solidali del territorio rivendicano che nella propria valle non ci sia gente in pericolo, in situazione di marginalità estrema, costretta a dormire per strada. L'ospitalità, in primo luogo, si inserisce in questa sensibilità di difesa del territorio in cui i cittadini vogliono avere voce in capitolo sulle scelte che riguardano la propria valle. Tale caratteristica di lotta di territorio contrasta con ciò che succede a Ventimiglia, a pochi chilometri di distanza, in cui la solidarietà dei locali è minoritaria - e legata soprattutto alla dimensione religiosa - e dove è più presente invece una solidarietà esogena di matrice politica. In quest'ultimo caso, si tratta di persone che militano nei movimenti, in particolare nell'esperienza dei centri sociali autogestiti, e arrivano da fuori (Milano, Bologna, Bergamo, Genova, ecc.) per un lavoro politico sulla critica alle frontiere. Se in Val Roja la solidarietà ai migranti assume una dimensione di causa territoriale ed è fondamentalmente di carattere endogeno, a Ventimiglia è invece più strettamente legata alla lotta politica contro i confini e più vicina ad una dimensione esogena (Giliberti, Queirolo Palmas, 2020).

L'azione solidale della Val Roja, che si configura nei termini di una risposta a una crisi territoriale - che in qualche modo obbliga gli abitanti a prendere posizione - presenta delle specificità legate al contesto rurale. Le caratteristiche di tale contesto facilitano la dimensione endogena: ci si conosce tutti, ci si incontra nel quotidiano nei paesi, è semplice coordinarsi e retroalimentare l'azione solidale attraverso la dimensione amicale. Le reti di solidarietà declinate al plurale, con diversi registri di azione, da Roya Citoyenne, a Défends ta Citoyenneté, che è dietro l'esperienza del campo di Breil, fino ai gruppi non mediatizzati (Giliberti, 2020) - sono in ogni caso tutte costituite da persone che hanno fatto una scelta di decrescita legata ad uno spirito comunitario. Una questione di valori, dunque, pensata nei termini della "difesa del territorio".

L'espressione "difesa del territorio" - al centro delle mobilitazioni che prendono forma nella valle - è scivolosa perché rivendicata da parti ideologiche opposte con prospettive di segno contrario. Da cosa e con quali obiettivi va difeso il territorio? Le risposte divergono notevolmente. Da un lato, nella prospettiva alternativa neorurale, la valle va difesa dalle politiche neoliberali dominanti che, seguendo Bourdieu (1998), producono un attacco alle strutture e alla dimensione collettiva per l'imposizione della logica del capitale, che genera sfruttamento intensivo e deturpazione del territorio. Dall'altro lato, la parte di segno opposto rivendica una difesa del territorio proprio contro l'azione dei movimenti alternativi cercando di preservarlo, per esempio, dall'accoglienza 
dei migranti negli ultimi anni. L'associazione Défendre la Roya il cui nome è emblematico rispetto al paradigma della difesa del territorio, vicina al Front National locale, nasce con l'obiettivo unico di ottenere la dissoluzione di Roya Citoyenne, accusata di banda organizzata finalizzata al favoreggiamento dell'immigrazione clandestina ${ }^{13}$.

Una parte degli abitanti esprime una posizione opposta alla solidarietà e alcuni contattano le forze dell'ordine quando avvistano un migrante sul territorio, oltreché denunciare chi aiuta ${ }^{14}$. In una visione vicina a quella dei discorsi anti-immigrazione di Eric Ciotti - influente politico locale repubblicano - o di Marine Le Pen, che ricordano quelli di Matteo Salvini o di Santiago Abascal, il punto di vista degli attori ostili all'aiuto dei migranti si muove sui luoghi comuni dell'estrema destra europea, in voga negli ultimi anni.

Chi sono questi migranti? Vengono qua per ottenere benefici sociali, per... rubare lo stato sociale ai francesi... vanno all'estero ma le loro mogli e i loro figli rimangono nel loro paese... è una vergogna... lasciano le loro famiglie nel loro paese e cosa fanno qua? E poi ci mettono in pericolo... nella valle abbiamo avuto problemi sanitari rispetto a tutti questi migranti che arrivano. Un mese e mezzo fa, è stato rilevato un virus all'asilo di Breil-sur-Roya. Cos'è questo virus? Giustamente, non lo sappiamo, ma sono cose che non avevamo mai visto prima. Ci sono studenti universitari che hanno preso la peste. Non l'avevamo mai avuta la peste qui (...) Non vogliamo un campo di migranti qui, geograficamente non c'entra nulla... cosa facciamo con tutti questi migranti qui? Abbiamo già tassi di disoccupazione molto elevati... constatiamo giorno dopo giorno il declino della Francia... (Alain, 56 anni, tecnico dello spettacolo)

In ogni caso sono vestiti meglio di me... hanno delle scarpe molto costose, lo smartphone di ultima generazione... e si approfittano dello Stato francese a discapito dei francesi... La Francia non ha capacità di accogliere tutti questi immigrati. E non capisco come facciano ad abbandonare la propria famiglia, il proprio paese, se ne fottono del proprio paese, della propria famiglia... e molti di loro non provengono da paesi che sono in guerra... (Toto, 63 anni, disoccupato)

Tali posizionamenti, contrari all'ospitalità, ostili ai migranti e al loro sostegno, che in certi casi denunciano l'azione dei solidali alla polizia, in generale sono vicini ai punti di vista conservatori delle famiglie native - le cosiddette familles de souche -, che rivendicano un'autoctonia esclusiva rispetto a chi è arrivato negli ultimi decenni e detengono una porzione rilevante del potere sui territori. Si generano dei conflitti all'interno della popolazione della valle, che rivelano delle frontiere sociali interne già esistenti, che l'irrompere della frontiera e delle sue conseguenze rafforzano. La frontiera socio-culturale tra coloro i quali si rivendicano nativi e coloro che sono arrivati nel corso degli ultimi decenni - i

${ }^{13}$ La domanda, presentata al Tribunale Amministrativo di Nizza, ha avuto esito negativo nel novembre del 2017.

${ }^{14}$ Uno dei processi giudiziari a cittadini della valle per favoreggiamento dell'immigrazione clandestina, denominato dei quattro papis et mamis - situato all'interno dei cosiddetti "delitti di solidarietà" - nasce da una segnalazione telefonica alla polizia di qualcuno che aveva visto i solidali partire con dei migranti per uno sterrato di montagna. 
neorurali, gli hippies secondo i nativi - subisce le conseguenze dirette della chiusura delle frontiere statali. La storica distanza tra autoctoni e neorurali costituisce così una faglia per la lettura del posizionamento degli abitanti della valle rispetto alla questione migratoria.

L'universo neorurale, che in linea generale si spende per la solidarietà ai migranti, interpreta i posizionamenti ostili ai migranti come principalmente frutto della paura, oltreché del razzismo dilagante negli ultimi anni.

L'aspetto principale per me è la paura. Al mercato, sento dei commenti in cui le persone esprimono immagini di paura, di orrore. Ho sentito gente dire: "Non possiamo più camminare di notte, perché sembra che ci siano dei neri che attraversano le montagne, è pericoloso...". Circolano discorsi del genere tra le persone. (Nadia, contadina, 37 anni)

Sicuramente c'è paura... e poi ci sono quelli che sono chiaramente razzisti e contro l'immigrazione... questa è una novità, quando ero piccola il Front National non esisteva nella valle... FN è una novità qui, è molto urbano come origine anche se oggi prende molti voti in campagna... nella valle il Front National ha 10 anni. (Christelle, attrice di teatro, 44 anni)

In ogni caso, in termini sotterranei, esiste una dimensione di porosità, in cui una parte, seppur minoritaria, dell'universo culturale souche fa dei timidi passi verso la dimensione di sostegno ai migranti. Si tratta di una rete invisibile, che agisce in modo insospettabile e nascosto non solo non rivendicato ma anche, in qualche modo, formalmente negato, in linea con il posizionamento formale del proprio gruppo di riferimento.

Tu questo non potrai mai saperlo, Luca... lo lo so perché sono di famiglia souche e perché si fidano di me... ma c'è una rete invisibile che chiama e mi dice: "ci sono persone lì sotto, vai a cercarle". Se tu vai a parlarci ti diranno che sono contrari... ma in ogni caso aiuteranno... e di questi tu non potrai sapere mai nulla... (Christelle, attrice di teatro, 44 anni)

Quando alcune famiglie souche aiutano i migranti rivolgendosi alla rete neorurale attiva, questa porosità, potenzialmente, sarebbe in qualche modo potuta sfociare in prove di riconciliazione. Al contrario, questo tipo di posizionamento rimane nascosto e negato, e ciò che si visibilizza è esclusivamente la contrarietà formale tra gli universi, che si dispongono l'uno contro I'altro. Utilizzando le categorie di Goffman (1997), nel retroscena una parte dell'universo souche partecipa - seppur marginalmente - alla solidarietà, ma sulla scena ciò che si visibilizza è un'acerrima opposizione al sostegno ai migranti. La crisi, sulla scena, si formalizza e una debole porosità, nel retroscena, viene negata e si soffoca progressivamente.

Si assiste così ad un'irrimediabilità della rottura pubblica tra universi, che caratterizza un dramma sociale (Turner, 1986) che prende forma nella valle, in cui l'opposizione tra gruppi si rafforza ed emerge la sfiducia reciproca verso l'integrità morale del gruppo contrapposto. 
È sicuro al $100 \%$ che hanno fatto del commercio con questa storia dei migranti. lo non credo che lo fanno per far del bene... ricevono soldi sui loro conti personali... E poi a questi migranti gli danno delle speranze per niente, li riportano subito in Italia... Sono andato a vedere il campo di Cédric Herrou, è una vergogna... Sono andato come testimone civile con la gendarmeria. Era un porcile. Questo non è un campeggio... è una discarica... è sporco, è disumano ricevere persone in quelle condizioni... C'erano 150/200 persone, c'erano escrementi ovunque... stanno meglio accampati a Ventimiglia che in quel posto inumano... Spetta al governo prendere in mano la situazione, non a Cédric Herrou... e lui ha 8-10 condanne... e continua a infrangere la legge, continuamente... cosa dovremmo pensare? Che è protetto? (Toto, 63 anni, disoccupato)

Défendre la Roya è stato il tentativo di fare qualcosa per questa valle che è in una fase di declino... Non c'è più quella piccola fiamma che c'era prima... i negozi chiudono, perché non vivono, vivono male. È un momento speciale questo... E i sindaci difendono chi aiuta i migranti, che sono degli approfittatori, che sono lì per benefici personali. (Alain, 56 anni, tecnico dello spettacolo)

\section{Note conclusive}

Dall'autunno 2017, pochissimi transitanti sono arrivati nella valle, per la crescente militarizzazione del territorio e le strategie di repressione portate avanti dalle forze dell'ordine. Inoltre, i solidali sono criminalizzati; in valle sono attorno ad una trentina le persone che sono state messe in custodia cautelare o sono state soggette a un controllo giudiziario e sono sei le persone della valle con processi in corso per favoreggiamento all'immigrazione clandestina ${ }^{15}$ (Giliberti, 2017; Gachet-Deuzeide, 2018). In questo contesto, per i transitanti non è più conveniente dirigersi verso la valle. La criminalizzazione della solidarietà sia nei termini dell'accusa di favoreggiamento, sia in relazione a penalità che incombono sull'attività assistenziale, come l'ordinanza contro la distribuzione del cibo - sottolinea la vulnerabilità delle reti e facilita una diminuzione della fiducia dei migranti in questa rotta migratoria. Tra i migranti, sembra diffondersi l'informazione secondo cui attraversare la Val Roja per entrare in Francia non sia più una tattica efficace: "No, in Roja è molto meglio non andare. Lì finisce male", mi dice un ragazzo eritreo bloccato a Ventimiglia nell'inverno del 2017.

Anche se da circa due anni la rotta che passa dalla Val Roja sembra essersi praticamente esaurita, la mobilitazione per i migranti continua ad avere una presenza latente e gli sporadici momenti di passaggio a cui aleatoriamente si

\footnotetext{
${ }^{15}$ Quando si parla in Francia di "délits de solidarité", ci si riferisce in primis all'accusa di "favoreggiamento dell'immigrazione clandestina"; l'espressione travalica questo capo d'imputazione della legislazione nazionale, associandosi per esempio ad ordinanze dell'amministrazione locale che vietano, per esempio, la distribuzione di beni di prima necessità ai migranti. In riferimento al favoreggiamento nella legislazione francese, il reato previsto dall'art. 622 del "Code de l'entrée et du sejour des étrangers et du droit d'asile" (Ceseda) punisce con pene fino a cinque anni di reclusione e 30.000 euro di multa "qualunque persona abbia, attraverso un aiuto diretto o indiretto, facilitato o tentato di facilitare l'ingresso, la circolazione o il soggiorno irregolare di uno straniero in Francia".
} 
assiste non può far escludere che, in futuro, la valle possa tornare ad essere teatro del passaggio dei migranti verso il nord Europa, oltreché di pratiche di ospitalità e di supporto al transito. In ogni caso, al blocco provvisorio di una rotta se ne aprono o si intensificano delle altre, come quella che dalla Val Susa entra nel Brianzonese. Esistente già dal 2016, questa rotta - sempre sulla frontiera franco-italiana - ha visto un aumento repentino del passaggio a partire dall'agosto 2017, in corrispondenza con la crescente diminuzione di quello in Val Roja. Tale rotta - che continua ad essere attiva e che presenta importanti reti solidali dall'una e dall'altra parte del confine (Bachellerie, 2019) - è in ogni caso più rischiosa e difficile, trattandosi di un territorio in alta quota, giungendo ai 1.762 metri d'altezza del Colle della Scala.

Da quando il passaggio in valle è residuale, Roya Citoyenne si è impegnata maggiormente nell'attività - che da sempre le caratterizza, in rete con associazioni nazionali e internazionali - di denuncia giuridica sulla violenza del confine, in collaborazione con un pool di avvocati, oltreché una lotta di carattere riformista-progressista affinché lo Stato rispetti le proprie leggi in relazione al diritto d'asilo e alla presa in carico dei minori non accompagnati. Una parte dei solidali continua le proprie attività di rivendicazione politica e giuridica, oltreché mediatiche e culturali ${ }^{16}$. Recentemente - dal luglio 2019 - sul terreno agricolo di Breil prende inoltre forma un nuovo progetto di accoglienza a lungo termine per alcune persone rimaste in valle. In continuità con l'esperienza del campo, ma modificandone la struttura di fondo, quest'esperienza costituisce la prima comunità agricola francese Emmaüs ${ }^{17}$. Il progetto propone I'agricoltura e la vita in campagna come mezzo per uscire dall'esclusione, in linea con i valori che gli abitanti solidali della valle difendono. Il progetto Emmaüs in versione agricola inaugurato da Cédric e compagni è una piccola esperienza di inclusione sociale, che in qualche modo si inserisce in continuità con il percorso di solidarietà della valle.

In una "crisi dell'accoglienza" (Lendaro, Rodier, Vertongen, 2019) che ad oggi non sembra risolversi, ma che si consolida, la mobilità migrante bloccata ai confini intraeuropei continua a generare le violente conseguenze che abbiamo analizzato nel corso dell'articolo. In ogni caso, la ricerca ci ha parimenti

${ }^{16}$ Un chiaro esempio in questo senso è il Festival estivo "Passeurs d'Humanité" (https:// passeursdhumanite.com/), già realizzato per due edizioni (2018 e 2019) - quando già il passaggio in valle era residuale -, organizzato durante quattro giorni nei paesini della valle dall'associazione "Les ami.e.s de la Roya" di Parigi, in collaborazione con i solidali del territorio. Evento moltitudinario che attira in valle centinaia di persone sui temi dell'ospitalità, ma anche dell'autodeterminazione alimentare e della sostenibilità rurale, propone conferenze di accademici e specialisti di vario tipo, tavole rotonde di dibattito collettivo, spettacoli teatrali e concerti.

17 Emmaüs è un movimento laico, fondato dall'Abbé Pierre, di contrasto alla povertà e all'esclusione, basato su gruppi di solidarietà che sviluppano attività economiche di vario tipo, scommettendo sulla re-inclusione sociale dei soggetti. 
mostrato il sorgere di una solidarietà dal basso, un'altra faccia della governance migratoria, una società civile che irrompe e partecipa a trovare risposte situate nei propri territori. In una valle rurale come la Val Roja, la solidarietà ai migranti in transito si genera così da una parte degli abitanti che, impegnati nella vita culturale e in una serie di mobilitazioni per il proprio territorio, cerca soluzioni alternative alle problematiche del nostro tempo e promuove nuovi modelli di società.

\section{Bibliografia}

AMBROSINI, Maurizio. Irregular Immigration in Southern Europe. Actors, Dynamics and Governance. Cham: Springer International Publishing, 2018.

Amnesty International. Des contrôles au confin du droit. Violations de droits humains à la frontière française avec l'Italie. 2017. Disponibile su : https://www. amnesty. $\mathrm{fr} /$ refugies-et-migrants/actualites/frontiere-franco-italienne-des-controles-auxfrontieres.

ANAFE. Persona non grata. Conséquences des politiques sécuritaires et migratoires à la frontière franco-italienne. Parigi: Anafé, 2019.

ANDERSON, Benedict. Comunità immaginate. Origini e diffusione dei nazionalismi. Roma: Laterza, 2018.

ARIS ESCARCENA, Juan Pablo. La paradoja del taxista: Ventimiglia como frontera selectiva. Mondi Migranti, n. 2, p. 99-114, 2018.

BABELS. Hospitalité en France: mobilisations intimes et politiques. Paris : Le passager clandestin, 2019.

BACHELLERIE, Sarah. Traquer et faire disparaitre les etrangers indesiderables. La fabrique de l'invisibilité à la frontière franco-italienne du Brianconnais. ENS de Lyon : Mémoire Master 2 - Sciences Sociales, 2019.

BALIBAR, Étienne. Europe as Borderland. Environment and Planning D Society and Space, v. 27, n. 2, p. 190-215, 2009.

BIREY, Tegije; CANTAT, Celine ; MACZYNSKA, Ewa; SEVININ, Eda. Challenging the Political Across Borders : Migrants' and Solidarity Struggles. Budapest : Center for Policy Studies, 2019.

BOURDIEU, Pierre. L'essence du néoliberalisme. Le Monde Diplomatique: 1998. Disponibile su: https://www.monde-diplomatique.fr/1998/03/BOURDIEU/3609.

COLLYER, Michael; DÜVELL, Franck; de HAAS, Hein. Critical Approaches to Transit Migration. Population, Space and Place, v. 18, n. 4, p. 407-414, 2012.

DÜVELL, Franck. Transit Migration: A Blurred and Politicised Concept. Population, Space and Place, v. 18, n. 4, p. 415-427, 2012.

DÜVELL, Franck; MOLODIKOVA, Irina N.; COLLYER, Michael (eds.). Transit Migration in Europe. Amsterdam: Amsterdam University Press, 2014.

FONER, Eric. Gateway To Freedom: The Hidden History of the Underground Railroad. New York: Norton, 2015. 
FONTANARI, Elena; BORRI, Giulia. Civil society on the edge: actions in support and against refugees in Italy and Germany. Mondi Migranti, n. 3, p. 23-51, 2017.

FREMEAUX, Isabelle; JORDAN, John. Les sentiers de I'utopie. Paris : La Découverte, 2012.

GACHET-DIEUZEIDE, Marion. Les conséquences du rétablissement des contrôles policiers à la frontière franco-italienne sud. Bruxelles: Les Verts-Ale / Les ami.e.s de la Roya, 2018.

GERBIER-AUBLANC, Marjorie. L'hybridation de I'humanitaire: des citoyens ordinaires dans les camps de migrants en France. Alternatives humanitaires, v. 9, p. 90-101, 2018.

GILIBERTI, Luca. La criminalizzazione della solidarietà ai migranti in Val Roja: note dal campo. Mondi Migranti, n. 3, p. 161-181, 2017.

GILIBERTI, Luca. Abitare la frontiera. Lotte neorurali e solidarietà ai migranti sul confine franco-italiano. Forthcoming. Verona: Ombre Corte, 2020.

GILIBERTI, Luca; QUEIROLO PALMAS, Luca. Solidarities in transit on the French/ Italian border. Ethnographic accounts from Ventimiglia and the Roya Valley. In: AMBROSINI, Maurizio; CINALLI, Manilo; JACOBSON, David (eds.). Migration, Borders and Citizenship. Between Policy and Public Spheres. Basingstoke: Palgrave Macmillan, 2020, p. 109-140.

Gisti-Cimade-Adde-Anafé . Contrôles frontaliers à Vintimille : référé liberté devant le Conseil d'Etat. 2015. Disponibile su: <https://www.gisti.org/spip. php?article4981>.

GOFFMAN, Erving. La vita quotidiana come rappresentazione. Bologna: Il Mulino, 1997.

HESS, Sabine. 'De-Naturalising Transit Migration. Theory and Methods of an Ethnographic Regime Analysis'. Population, Space and Place, v. 18, n. 4, p. 428240, 2012..

KYMLICKA, Will. Solidarity in diverse societies: beyond neoliberal multiculturalism and welfare chauvinism. Comparative Migration Studies, v. 3, p. 1-19, 2015.

LATOUCHE, Serge. Come si esce dalla società dei consumi. Corsi e percorsi della decrescita. Torino: Bollati Boringhieri, 2011.

LENDARO, Annalisa. Désobéir en faveur des migrants. Répertoires d'action à la frontière franco-italienne. Journal des anthropologues, n. 152-153, p. 171-192, 2018.

LENDARO, Annalisa; RODIER, Claire; VERTONGEN, Yuti L. La crise de l'accueil. Frontières, droits, rèsistances. Paris : La Dècouverte, 2019.

LOPERFIDO, Giacomo. Integralismo culturale e xenofobia. In: RICCIO, B. (a cura di) Antropologia e migrazioni. Roma: Cisu, 2017, p. 121-132.

MARTINI, Francesca; PALIDDA, Salvatore. Continuità e mutamenti delle migrazioni nel confine tra I'Italia e la Francia. Altreitalie, v. 56, n. 2, p. 117-129, 2018. 
MOLLARD, Chloë. L'accueil des migrants dans la vallée de la Roya: Hospitalité privée en contexte d'inhospitalité politique. Université Nice Sophia Antipolis: Mémoire de Master 1 - Sociologie, 2017.

NOGUÉ, Joan. El reencuentro con el lugar: nuevas ruralidades, nuevos paisajes y cambio de paradigma. Documents d'Anàlisi Geogràfica, v. 62, n. 3, p. 489-502, 2016.

QUEIROLO PALMAS, Luca. Nuit debout. Transiti, connessioni e contestazioni negli accampamenti urbani dei rifugiati a Parigi. Mondi Migranti, v. 32, n. 2, p. 207-227, 2017.

QUEIROLO PALMAS, Luca; RAHOLA, Federico. Underground Europe. Lungo le rotte migranti. Fortcoming. Milano: Meltemi, 2020.

QUEIROLO PALMAS, Luca; STAGI, Luisa. Dopo la rivoluzione. Paesaggi giovanili e sguardi di genere nella Tunisia contemporanea. Verona: Ombre Corte, 2017.

RAHBI, Pierre. La parte del colibrì. La specie umana e il suo futuro. Torino: Lindau Edizioni, 2014.

REA, Andrea; MARTINIELLO, Marco; MAZZOLA, Alessandro; MEULEMAN, Bart. The refugee reception crisis in Europe. Polarized opinions and mobilizations. Bruxelles : Editions de l'Université de Bruxelles, 2019.

RRDP - Refugee Rights Data Project. In dangerous transit. Filling information gaps relating to refugees and displaced people in Ventimiglia, Italy. London: RRDP Refugee Rights Data Project, 2017.

STREIFF-FÉNART, Jocelyne; POUTIGNAT, Philippe. Nouadhibou « ville de transit »? Le rapport d'une ville à ses étrangers dans le contexte des politiques de contrôle des frontières de l'Europe. Revue européenne des migrations internationales, v. 24, n. 2, p. 193-217, 2008.

TURNER, Victor. Dal rito al teatro. Bologna: Il Mulino, 1986. 